\title{
AUTISMO: DESCOBERTA TARDIA, IMPORTÂNCIA DA TERAPIA COGNITIVO COMPORTAMENTAL NA INTERVENÇÃO PSICOTERAPÊUTICA
}

\section{ARTIGO DE REVISÃO}

LOBATO, Makatiney De Farias ${ }^{1}$

MARTINS, Maria das Graças Teles ${ }^{2}$

LOBATO, Makatiney De Farias. MARTINS, Maria das Graças Teles. Autismo: Descoberta tardia, importância da terapia cognitivo comportamental na intervenção psicoterapêutica. Revista Científica Multidisciplinar Núcleo do Conhecimento. Ano 05, Ed. 12, Vol. 02, pp. 88-105. Dezembro de 2020. ISSN: 24480959, Link de acesso:https://www.nucleodoconhecimento.com.br/semcategoria/descoberta-tardia

\section{RESUMO}

Introdução: O transtorno do Espectro Autista (TEA) tem início precoce e de curso crônico com um conjunto de distúrbios da socialização. Manifesta-se antes dos três anos de idade sendo considerado por anomalias que atingem as esferas da interação social, comunicação e comportamento que se apresenta restrito e repetitivo. Diferentes literaturas explicitam que o diagnóstico e a aplicação precoce de intervenções terapêuticas são essenciais para a reabilitação global e a promoção de

\footnotetext{
${ }^{1}$ Bacharel em Psicologia.

2 Orientadora. Formação em Psicologia - Centro Universitário de João Pessoa UNIPÊ (2000), Especialização em Psicologia da Saúde Desenvolvimento e Hospitalização (UFRN-2003/2004), especialização em sexualidade humana (UFPB-2001/2002). Mestrado em Ciências da Educação - Universidade Lusófona de Humanidades e Tecnologias - ULHT- Portugal (2005), reconhecido pela UFBA (28.10.2009). Mestrado em Saúde Coletiva - UNIFESP (2010).
} 
uma qualidade de vida da pessoa autista. Objetivo: Compreender o Transtorno do Espectro Autista (TEA), história, características, prejuízos e diagnóstico tardio e apresentar as contribuições interventivas da Terapia Cognitivo Comportamental no tratamento psicoterapêutico. Problema: Como a Terapia Cognitivo Comportamental contribui no tratamento interventivo psicoterapêutico de pacientes autistas com diagnostico tardio? Metodologia: A pesquisa é de cunho bibliográfica exploratória, baseada em revisões literárias retirados de sites e livros que abordam essa temática. Resultados: A Terapia Cognitivo Comportamental é uma das terapias mais bem desenvolvida, confiável e eficaz no atendimento da pessoa com autismo, justamente porque tem uma abordagem flexível, adaptável às mudanças que ocorrem no nível de desenvolvimento, promovendo um modelo específico de tratamento terapêutico, o qual consiste em: aquisição, fluência, manutenção, generalização e adaptação, e assim, cada item pode ser adequado para atender as finalidades do grau de aprendizagem do autista, salienta-se que esta abordagem é feita de maneira responsável e direcionada é possível que o comportamento da pessoa diagnosticada tardiamente com autismo obtenha mudanças ainda que mínimas mas que se configuram como um grande passo para a melhoria na qualidade de vida da pessoa com TEA, proporcionando assim uma melhor adaptação na sociedade e estímulo para o desenvolvimento da comunicação, visando a autonomia e independência desses indivíduos.

Palavras-Chaves: Transtorno do Espectro Autista, terapia cognitivo comportamental, diagnóstico tardio.

\section{INTRODUÇÃO}

O objetivo do presente estudo é compreender o Transtorno do Espectro Autista (TEA), história, características, prejuízos e diagnóstico tardio em adultos e apresentar as contribuições interventivas da Terapia Cognitivo Comportamental no tratamento psicoterapêutico.

O transtorno do Espectro Autista (TEA) tem início precoce e de curso crônico com um conjunto de distúrbios da socialização. Manifesta-se antes dos três anos de idade 
sendo considerado por anomalias que atingem as esferas da interação social, comunicação e comportamento que se apresenta restrito e repetitivo. Diferentes literaturas explicitam que o diagnóstico e a aplicação precoce de intervenções terapêuticas são essenciais para a reabilitação global e a promoção de uma qualidade de vida da pessoa autista.

As motivações que conduziram a este tema partiram do princípio em compreender de que maneira o autismo tem agravado a vida de pessoas adultas que não foram diagnosticados precocemente e de que maneira a psicologia tem trabalhado com essas pessoas intuindo amenizar o comprometimento das principais áreas do desenvolvimento humano.

Este estudo se justifica em razão de ser um tema no qual ainda existem poucas produções uma vez que grande parte das literaturas abordam o autismo na infância, enquanto que o autismo diagnosticado tardiamente não tem sido muito discutido apesar de causar um comprometimento mais acentuado na vida desses indivíduos na fase adulta, nesse segmento entende-se que a terapia cognitivo comportamental pode trazer benefícios no auxílio ao tratamento dessas pessoas visto que a TCC é flexível e pode ser usada para os mais diversos tratamentos, dentre eles o Autismo tardio.

Diante do exposto, estabeleceu-se a hipótese de que a pessoa com diagnóstico tardio teria maiores comprometimentos visto que os mecanismos de desenvolvimento do cérebro já estão formados, deste modo, presumiu-se que, os resultados a longo prazo poderiam ser mais complexos. Estimou-se que com a intervenção da Terapia Cognitivo Comportamental (TCC) poderia haver resultados significativos com a estimulação através das técnicas intuindo os ganhos no desenvolvimento das habilidades de socialização e comunicação reduzindo os sintomas e os prejuízos de desenvolvimento gerados pelo transtorno. Buscou-se, ainda, apresentar o contexto histórico do autismo, conceito e características, identificar os prejuízos provocados pelo diagnóstico tardio do TEA em adultos, e descrever as contribuições interventivas da terapia cognitivo comportamental para o quadro complexo do autismo em adultos. $\mathrm{Na}$ classificação de transtornos mentais e de comportamento proposta pelo código 
internacional de doenças - CID 10, o autismo é classificado na categoria dos transtornos invasivos do desenvolvimento (OMS, 1996).

Em consonância com essa instituição, o transtorno do Espectro Autista é assinalado por anomalias qualitativas em três áreas importantes que são a interação social, comunicação e comportamento que se mostra restrito e repetitivo. Um outro fator importante de ser registrado é que a quinta versão do Manual Diagnóstico e Estatístico de Transtornos Mentais - DSM-V (APA, 2013) esclarece que o diagnóstico do TEA deve basear-se em acordo com os seguintes critérios: a) déficit persistente na comunicação e na interação em vários contextos, não relacionados a atraso do desenvolvimento; b) ausência ou dificuldade na reciprocidade emocional e social; c) aproximação social anormal, falha na conversação e compartilhamento de interesses reduzido; d) reprodução de falha na demonstração de emoções e afeto; e) problemas para iniciar uma interação social.

Sendo assim, este estudo buscou responder a seguinte questão problema: Como a Terapia Cognitivo Comportamental contribui no tratamento interventivo psicoterapêutico de pacientes autistas com diagnostico tardio?

Destaca-se que a Terapia Cognitivo Comportamental apresenta estratégias e técnicas interventivas cognitivas e comportamentais que podem ser aplicadas com os autistas, dentre elas estão, a intervenção intensiva onde podem ser incluídas atividades rotineiras caseiras como tomar banho ou alimentar-se e em comportamentos complexos como aquisição de comportamento de comunicação e interação social, a automonitoração na qual a pessoa aprende a definir seus próprios sentimentos e pensamentos e saber comunica-los às pessoas. Possibilita diminuir o comportamento de frustração, de ansiedade e agressividade.

Espera-se com este estudo evidenciar conhecimentos sobre o autismo e as suas implicações em casos diagnosticados tardiamente não só apenas no campo da psicologia, mas também seja de relevância para a sociedade no geral e principalmente para os adultos autistas que por algum motivo não puderam ser diagnosticados na infância. 


\section{METODOLOGIA}

Este estudo foi amparado no aporte metodológico de revisão da literatura com o método de pesquisa bibliográfica e exploratória. Gil (2017) demonstra que a pesquisa bibliográfica é compreendida como sendo a leitura e compressão de trabalhos já publicado para explicar ou procurar resposta de um problema ou hipótese. Os materiais utilizados foram: livros, e artigos científicos disponíveis nas plataformas da Scientific Electronic Library Online (Scielo), Biblioteca virtual em saúde (BV-Saúde), Periódicos eletrônicos em Psicologia (Pepsic) publicados no período de 2010 a 2019.

Cervo e Bervian (2013) afirma que a pesquisa exploratória define objetivos e busca mais informações sobre o assunto estudado e realiza descrições precisas da situação para descobrir as relações existentes entre seus elementos componentes. Os critérios de inclusão e exclusão se relacionam a conteúdos pertinentes ao tema proposto e que contenham as palavras chaves: Transtorno do Espectro Autista. Terapia cognitivo comportamental. Diagnóstico tardio. Foram descartados os materiais que não se enquadram na temática em questão. Concernente a análise crítica dos riscos e benefícios, em razão deste trabalho ser de cunho bibliográfico não foi utilizado o Termo de Consentimento Livre e Esclarecido (TCLE).

\section{CONTEXTO HISTÓRICO SOBRE O AUTISMO, CONCEITO E CARACTERÍSTICAS}

Os primeiros diagnósticos sobre autismo foram feitos há mais de meio século e ainda hoje, 2020 é uma incógnita para profissionais que buscam respostas e tratamentos para esse transtorno. O termo autismo foi utilizado pela primeira vez pelo psiquiatra austríaco Eugen Bleuler em 1911 para se referir a um dos critérios adotados em sua época para a realização de um diagnóstico de Esquizofrenia, desse modo percebe-se que antes dos estudos sobre autismo, a pessoa com esse transtorno era tida como esquizofrênica. (LINHARES, 2012).

Apesar do termo autista ser utilizado por Eugen Bleuler como explicado nos parágrafos acima supracitados, o diagnóstico de uma pessoa considerada autista se 
deu pela primeira vez em 1943, sob os cuidados do médico Léo Kanner, no qual naquela ocasião observou e avaliou 11 crianças (KANNER, 1966, p. 720, apud ANDRADE, 2018, p. 17).

Nesse exposto Kanner, constatou que a criança autista apresentava características fora dos padrões consideradas normais visto que não possuíam habilidades comportamentais concernente as relações sociais, outrossim demonstravam dificuldades na comunicação e imaginação, características que mais tarde foram utilizadas como a "tríade" para o diagnóstico do autismo.

Outro nome que receberia décadas depois grande notoriedade quanto a seus estudos com crianças autistas foi Hans Asperger,1944, quando expôs alguns quadros semelhantes ao autismo, quadros esses que apresentavam perturbações afetivas. (GUPTA et. al, 2006). Somente nos anos de 1980 é que o trabalho de Asperger foi traduzido para o inglês possibilitando novas descobertas sobre o transtorno. Contudo nos estudos de Asperger as crianças que foram estudadas por ele, não apresentaram a ecolalia como um problema linguístico, mas preferiam o isolamento a interação. (BAPTISTA e BOSA, 2002).

De acordo com Baptista e Bosa (2002) percebe-se que por muito tempo o autismo foi considerado como psicose, mas essa compreensão mudou conforme evoluíram os estudos como pode ser observado:

As primeiras edições do CID não fazem qualquer menção ao autismo. A oitava edição o traz como uma forma de esquizofrenia, e a norma agrupa-o como psicose infantil. A partir da década de 80 , assiste-se a uma verdadeira revolução paradigmática no conceito, sendo o autismo retirado da categoria de psicose no DSM-III e no DSM-III-R, bem como na CID-10, passando a fazer partes dos transtornos globais do desenvolvimento (BAPTISTA e BOSA, 2002, p. 28).

Com base na percepção dos autores supracitados, percebe-se o quanto foi complexo entendimento sobre o autismo para que assim o indivíduo com esse transtorno pudesse ser assistido de maneira plena, a ausência de estudos mais aprofundados sobre o autismo por muitos anos permitiram que autistas fossem tidos como pessoas esquizofrênicas, doentes mentais entre outras atribuições e nomenclaturas. 
O autismo foi incluído em 1968 no Manual Diagnóstico e Estatístico de Transtornos Mentais (DSM-II) na categoria de esquizofrenia de início da infância, mas a partir da década de 1980 foi retirado da classificação das psicoses e incluído no DSM-III como distúrbio invasivo do desenvolvimento (DIAS, 2015). No entanto só no DSM-IV, em 1994, o autismo passou a ser considerado parte dos distúrbios globais do desenvolvimento, juntamente com outros como; a Síndrome de Asperger e a Síndrome de Rett. (AMERICAN PSYCHIATRIC ASSOCIATION, 2013).

Segundo o DSM-V (2014), o autismo está posto como transtorno do espectro autista em função da imensa variedade de características que os autistas apresentam sendo diferentes entre si. Via de regra, compreende-se que cada autista é único, portanto, não se deve dizer que a pessoa com autismo faz isso ou faz aquilo, deste ou daquele jeito, algumas pessoas com autismo não falam, outras não param de falar. (LOVAAS, 2010). Ainda de acordo com este pesquisador, existem alguns indivíduos que se auto machucam, ou machucam outras pessoas, ou não machucam ninguém. Assim como, muitas pessoas autistas são apegadas, de modo extremo, a rotinas rígidas, enquanto outras não ligam para isso.

NO CID 10, o autismo está classificado nos Transtornos Globais do Desenvolvimento, enquanto que no, CID 11, que ainda não está em vigência, mas já foi lançado para que as instituições comecem tomar conhecimento sobre este documento, o autismo está classificado como "Distúrbio do Desenvolvimento Neurológico" e posto da seguinte maneira de acordo o site da Organização Pan-Americana de Saúde (ORGANIZAÇÃO PAN-AMERICANA DA SAÚDE, 2018).

6A02.0 - Transtorno do Espectro do Autismo sem deficiência intelectual (DI) e com comprometimento leve ou ausente da linguagem funcional;

6A02.1 - Transtorno do Espectro do Autismo com deficiência intelectual (DI) e com comprometimento leve ou ausente da linguagem funcional;

6A02.2 - Transtorno do Espectro do Autismo sem deficiência intelectual (DI) e com linguagem funcional prejudicada;

6A02.3 - Transtorno do Espectro do Autismo com deficiência intelectual (DI) e com linguagem funcional prejudicada; 
6A02.4 - Transtorno do Espectro do Autismo sem deficiência intelectual (DI) e com ausência de linguagem funcional;

6A02.5 - Transtorno do Espectro do Autismo com deficiência intelectual (DI) e com ausência de linguagem funcional;

6A02.Y - Outro Transtorno do Espectro do Autismo especificado;

6A02.Z - Transtorno do Espectro do Autismo, não especificado. (CID 10, p. 246).

Destarte, a nova CID-11 unificou todos os diagnósticos de Transtornos do Espectro do Autismo, classificando as diferentes formas que o autismo apresenta nos indivíduos, porém levou em consideração a deficiência intelectual e a linguagem funcional. O documento teve como base a alteração feita em 2013 na nova versão do Diagnostic and Statistical Manual of Mental Disorders, o DSM-5, que reuniu todos os transtornos que estavam dentro do espectro do autismo num só diagnóstico: TEA.

O autismo se caracteriza pelo desenvolvimento lento ou alterado no qual a pessoa com esse transtorno apresenta perturbações comportamentais de caráter afetivo, emocional e cognitivo, tendo prejuízos nas interações sociais, comunicação, apresentando comportamento focalizado e repetitivo. (BARON COHEN; LAI, 2015). O Transtorno do Espectro Autista (TEA), popularmente conhecido como autismo, se caracteriza por um quadro clínico, não há exames específicos como exame de sangue, mapeamento cerebral ou ressonâncias, que vão diagnosticar o sujeito com esse tipo de transtorno. (BARON COHEN e LAI, 2015).

Deve-se dizer que os indivíduos com TEA podem apresentar diferenças entre si. $\mathrm{O}$ autismo é um distúrbio muito comum quando levado em consideração as últimas estatísticas do CDC (Centro de Controle de Doenças e Prevenção do governo dos EUA, 2014) que apontam um número significativo de diagnósticos de autismo na atualidade, de acordo com este órgão para cada 59 pessoas uma é diagnosticada com autismo. Em conformidade com Beck et al., (2014), para que o indivíduo seja considerado autista é preciso que ele apresente prejuízos em três primordiais áreas do desenvolvimento humano: comunicação, comportamento e interação social. 
Na perspectiva de Fonseca (2009), pessoas autistas poderão apresentar ecolalia, ou seja, a repetição de algo que alguém acabou de dizer, incluindo palavras, expressões ou diálogos. De acordo com as literaturas revistas entende-se que apesar de existirem consensos entre especialistas em relação à presença de possíveis alterações no sistema nervoso central que levariam a uma desordem no padrão de desenvolvimento da pessoa autista conforme assegura Mello (2007), não é possível determinar qualquer aspecto biológico e/ou ambiental que contribua de forma decisiva para que ocorram as manifestações desse transtorno.

Sendo assim, salienta-se que as causas específicas e definitivas capazes de explicar o autismo ainda não foram identificadas. Estudos apontam que o transtorno atinge em predominância o sexo masculino, estimando cerca de três a quatro meninos afetados para uma menina. No entanto, as meninas tendem a ser mais comprometidas com seriedade quando afetadas. (SILVA; MULICK, 2009).

Encontrou-se também nas literaturas alguns estudos realizados por Baio et al., (2014) e Christensen, Baio, Braun, (2016) no qual por meio de investigações estimaram um aumento drástico de casos, atingindo em média 40 a 60 casos a cada 10.000 nascimentos. Estudos anteriores, realizados em 2012 com crianças de 8 anos de idade nos Estados Unidos, apontaram uma prevalência global de 14,6 por 1.000 ou 1 em 68 crianças (BAIO et al., 2013).

Conforme pode ser visto, os estudos com crianças são os que prevalecem nas literaturas, entretanto nas pessoas autistas que não puderam ser diagnosticadas na infância e se tornaram jovens, adultos no qual suas famílias não puderam saber qual a causa para o isolamento, falta de comunicação e interação social, o comprometimento das interações tende a ser maior.

\subsection{OS PREJUÍZOS CONCERNENTES AO AUTISMO DIAGNOSTICADO TARDIAMENTE}

Entende-se que o autismo é um transtorno que tem causado muitas especulações acerca do comprometimento e implicações na vida cotidiana de sujeitos com TEA - 
Transtorno do Espectro do Autismo. Desse modo, tem sido comum as recomendações de especialistas e profissionais da área sobre a importância do diagnóstico precoce para que o tratamento e intervenções possam surtir efeitos positivos na vida de pessoas autistas.

Todavia, considerando que este é um transtorno relativamente novo no qual as pesquisas existentes tendem a debruçar-se sobre o transtorno na infância, pouco se sabe sobre como vivem as pessoas jovens ou adultas com diagnostico tardio. "No adulto, ao contrário do que tem vindo a acontecer na população infantil, não têm sido tão extensamente estudadas, o que se reflete em dificuldades no diagnóstico e tratamento nesta faixa etária" (RAMOS et al., 2014, p. 36).

No entendimento deste autor, com o passar dos anos a pessoa autista pode apresentar alguns progressos concernentes a convivência social e a comunicação desde que seja estimulada na infância. Fecteau (2017) argumenta que, a maioria dos adultos com TEA vive uma vida completamente dependente, sem autonomia alguma.

Em conformidade com os estudos realizados pelos pesquisadores acima citados, menos de um terço tem emprego regular; a maioria vive com os pais ou vive com apoio; e aqueles que estão empregados geralmente estão em empregos que pagam abaixo de um salário digno. Na mesma linha de pesquisa Seltzer (2017) chama a atenção para o fato em que devido a esses altos níveis de dependência, a maioria dos indivíduos com TEA precisará de algum tipo de apoio ou intervenção, geralmente em níveis intensos, durante a adolescência e a idade adulta.

O autor Gádia (2014) esclarece que nos adultos os déficits de linguagem são comuns, dentre os quais, uma quantidade significativa de autistas permanecem não-verbais. Deste modo, quanto aos que adquirem habilidades verbais, em suma, podem demonstrar déficits constantes no ato da conversação, como a falta de reciprocidade, dificuldades na compreensão das sutilezas de linguagem, piadas ou sarcasmo, assim como problemas na interpretação de linguagens corporais e expressões faciais. 
Muitas vezes na infância o autismo passa despercebido, tornando-se mais evidente no final da infância. $\mathrm{Na}$ adolescência e fase adulta as peculiaridades tendem a ter oscilações, nos relacionamentos há a falta de empatia, não conseguem captar as emoções de seus respectivos parceiros, causam estranheza nos ambientes em que fazem parte, são considerados pessoas anti sociais, isoladas em seu 'próprio mundo. (BRITES, 2019).

Por outro lado, no adulto autista, há uma melhora na adaptação a mudanças, porém "os interesses restritos persistem, e aqueles com habilidades cognitivas adequadas tendem a concentrar seus interesses em tópicos limitados, tais como horários de trens/aviões, mapas ou fatos históricos, etc., os quais dominam suas vidas" (ROBERTSON, 2015, p.23).

Nos adultos um dos prejuízos que podem ser identificados é o comportamento anti social, dificuldades de compreender discursos e dar retornos em linguagens não verbais, dificilmente adultos autistas escondem o que pensam, possuem linguagem direta, não se preocupando se vão afetar alguém com suas palavras. (GÁDIA, 2014).

\section{RESULTADOS E DISCUSSÃO}

\subsection{CONTRIBUIÇÕES INTERVENTIVAS DA TERAPIA COGNITIVO COMPORTAMENTAL EM PESSOAS COM TEA DIGNOSTICADO TARDIAMENTE}

Considerando todas as necessidades específicas do autismo, a psicologia tem apresentado diversas maneiras que podem corroborar em um melhor desempenho de pessoas com TEA mesmo na fase adulta. Nesse exposto, tem-se a Terapia Cognitivo Comportamental, desenvolvida por Aaron Beck no início da década de 1960, uma forma de psicoterapia cientificamente testada e tida como efetiva em mais de 300 pesquisas clínicas para diversos tipos de transtornos. "É uma terapia voltada para a resolução de problemas do paciente, geralmente é breve e tem eficácia científica e experimental" (SANTOS, 2017, p. 30). 
Moniz (2010), assevera que os maiores precursores da prática clínica e educativa comportamental foram Wolpe (1952), Eysenck $(1959,1960)$ e Skinner (1938). Porém é a Skinner e seus discípulos Lindsley, e Salamon, (1953) que se deve o termo modificação do comportamento. White et al., (2013) investigaram o efeito da TCC nas habilidades sociais em pessoas adultas com TEA e os resultados apontaram êxito no maior desempenho da comunicação.

Do mesmo modo, o estudo realizado por Hesselmark et al (2013) apontou que a Terapia Cognitivo Comportamental traz uma configuração de estrutura e previsibilidade importante para pessoas com TEA. As técnicas de definição de metas, tarefas de casa e a prática de habilidades sociais, assim como outras técnicas convencionais da TCC são apontadas como adequadas para o auxílio ao tratamento dessas pessoas. Lima e Dilascio (2016) explanam como a TCC proporciona ao indivíduo adulto com TEA uma melhora na consciência social, nas estratégias sociais, na qualidade de vida e na redução de sintomas psiquiátricos.

Destaca-se que a Terapia Cognitivo Comportamental se concentra em manipulação de comportamentos e variáveis das pessoas, na crença de que todo comportamento, tanto os adequados quanto os inadequados são aprendidos. Este mesmo autor, acima supracitado, afirma que a terapia comportamental entende que o paciente é único, e seus problemas são produto de uma história particular. "Isso humaniza o processo de terapia, pois se busca entender cada paciente e cada história, antes de propor qualquer intervenção". (SILVEIRA, 2015, p. 19).

Para Bahls e Navolar, (2010), as técnicas e finalidades conceituais da Terapia Cognitivo Comportamental - TCC, provém de duas principais abordagens: a cognitiva e a comportamental, as quais são analisadas no contexto do movimento integrador na psicologia que culminou no que atualmente denominam-se de terapias cognitivocomportamentais. A Terapia Comportamental se baseia no behaviorismo, é efetiva na redução de sintomas e taxas de recorrência, com ou sem medicação, em uma ampla variedade de transtornos psiquiátricos (KNAPP; BECK, 2011).

Na compreensão de Borges (2013) as técnicas mais usuais para a intervenção são: 
- Psicoeducação propicia uma maneira de auxiliar o tratamento das doenças mentais segundo as mudanças comportamentais, sociais e emocionais, permitindo, assim, a prevenção na saúde. Deste modo, a psicoterapia trata de um processo com caráter educativo aplicado tanto aos pacientes quanto aos seus respectivos cuidadores, objetivando ensiná-los a respeito do seu tratamento psicoterápico, de modo conscientizá-los e prepará-los para lidar com as mudanças a partir das estratégias de enfrentamento, do fortalecimento da comunicação e da adaptação (BHATTACHARJEE et al., 2016).

- A psicoeducação é uma das mais relevantes técnicas utilizadas na terapia cognitivo-comportamental, visto que pode-se potencializar a sua eficácia, por meio desta intervenção pode-se instrumentalizar o paciente no seu processo de mudança, nesse viés, quando esta técnica é empregada as chances para que o paciente possa adquirir autonomia são expressivas dado o processo terapêutico que com que o paciente sinta-se capaz de identificar e mudar pensamentos, manejar emoções e quebrar padrões de comportamentos que não o ajudam. (AGUIAR, 2018).

- ABA (Applied Behavior Analysis), é uma terapiaque se concentra na melhoria de comportamentos específicos, como habilidades sociais, comunicação, leitura e habilidades acadêmicas, além de habilidades de aprendizado adaptável, como destreza motora fina, higiene, cuidados domésticos, pontualidade e competência profissional. (REICHOW, 2012). Um dos métodos da ABA consiste no chamado "Treino por Tentativas Discretas", nesta proposta a aprendizagem se dá em divisões, ensinados por etapa, uma de cada vez durante uma série de tentativas. "Este procedimento é repetido várias vezes até o comportamento ser adquirido e emitido independente da ajuda física" (BORBA e BARROS 2018, p.13).

- PECS (Picture Exchange Communication System) trata-se de um sistema de comunicação frequentemente utilizado em indivíduos com autismo e/ou pouca fala funcional. (MIZAEL, 2013);

- TEACCH (Treatment and Educatin of Autistic and Related) - é um programa que envolve as esferas de atendimento educacional e clínico, em uma prática com abordagem psicoeducativa, (KWEE, 2018); 
- Método Miller - este método busca desenvolver o comportamento funcional por meio de sistemas lúdicos ao passo em que estimula a linguagem oral acompanhada pela linguagem de sinais. (SERRA, 2014);

- Realidade Virtual e Estimulação Cognitiva - é a interface entre o usuário e a simulação computadorizada em um determinado ambiente, oferece interações através de múltiplos canais sensoriais encontrados em vídeo games, e computadores no geral, oferecendo ao indivíduo a possiblidade de vivenciar emoções diversas. (CAIANA, 2016);

Em virtude das diversas técnicas utilizadas pela TCC para o melhor desenvolvimento do adulto autista percebe-se que esta terapia é considerada por muitos pesquisadores a mais bem desenvolvida, confiável e eficaz no atendimento da pessoa com autismo, por se tratar de uma abordagem flexível, adaptável às mudanças que ocorrem no nível de desenvolvimento. (GAUDERER, 2010).

Soares (2012) afirma que a aplicabilidade do TCC em pacientes com TEA promove um exemplar específico de tratamento terapêutico que incide na aquisição, na fluência, na manutenção, na generalização e na adaptação, podendo cada item ser adequado, a fim de atender os desígnios do grau de aprendizagem do autista.

Além dessas técnicas, a Terapia Cognitivo Comportamental apresenta outras estratégias que podem ser aplicadas para pessoas com autismo, dentre elas destacam-se:

- Intervenção intensiva onde podem ser incluídas atividades rotineiras caseiras como tomar banho ou alimentar-se e em comportamentos complexos como aquisição de comportamento de comunicação e interação social, vem ganhando atenção por sua eficácia demonstrada na promoção do engajamento social em indivíduos com graves deficiências comunicativas. (ZEEDYK et al., 2010)

- A automonitoração, na qual a pessoa aprende a definir seus próprios sentimentos e pensamentos e saber comunicá-los às pessoas possibilita diminuir o comportamento de frustração, de ansiedade e agressividade, a 
técnica de autoinstrução é empregada para que a pessoa possa orientar seu próprio comportamento. Sampaio (2005, p. 2), afirma "A técnica consiste em ensinar a criança como orientar seu próprio comportamento, servindo o terapeuta como o modelo a ser seguido pela criança no seu aprendizado".

Nesse modelo, o terapeuta realiza uma atividade relativamente simples, orienta verbalmente, em voz alta, todos os passos de sua ação. Posteriormente a pessoa é motivada a realizar a mesma atividade orientada pela fala do terapeuta. Em seguida a pessoa autista assume a responsabilidade de dirigir sua atividade, falando em voz alta os passos que deve seguir (autoinstrução). Nesta perspectiva compreende-se que quando a abordagem é feita de maneira responsável e direcionada é possível que o comportamento da pessoa diagnosticada tardiamente com autismo obtenha mudanças ainda que mínimas mas que se configuram como um grande passo para a melhoria na qualidade de vida da pessoa com TEA, proporcionando assim uma melhor adaptação na sociedade e estímulo para o desenvolvimento da comunicação, visando a autonomia e independência dessas pessoas.

Vale ressaltar que a TCC trabalha no contexto do autismo tratando determinados sintomas, como os de ansiedade, através de uma gradativa exposição aos estímulos aversivos, onde os pacientes são inibidos de lançar mão de táticas, a fim de reduzir a ansiedade até que a mesma tenha se diminuído naturalmente (UNG et al., 2015).

\section{CONCLUSÃO}

Neste estudo buscou-se compreender o Transtorno do Espectro Autista (TEA), história, características, prejuízos e diagnóstico tardio em adultos e apresentar as contribuições interventivas da Terapia Cognitivo Comportamental no tratamento psicoterapêutico.

Observou-se que o autismo é um transtorno que atinge as principais áreas do desenvolvimento humano, considerou-se relevante compreender quais as principais características dessa síndrome, e como as pessoas com diagnostico tardio poderiam 
ser afetadas, e de que forma a Terapia Cognitivo Comportamental, poderia auxilia-los nesse contexto.

Levando em consideração as análises das literaturas dispostas e utilizadas nesse trabalho, averiguou-se que o problema da pesquisa foi respondido haja visto que constou-se que a TCC pode auxiliar as pessoas com diagnostico tardio no sentido de direciona-los, quanto ao auto cuidado, propiciar funcionalidade social, preparando as pessoas com autismo para ter autonomia e assim reduzir suas dificuldades, intuindo a redução de ansiedade e dependência de familiares e cuidadores. Os objetivos traçados foram alcançados quando por meio de revisões bibliográficas, compreendeuse o que é o autismo, quais as implicações na vida da pessoa autista diagnosticada tardiamente e que existem possiblidades de intervenção da TCC.

Nessa perspectiva, as hipóteses foram confirmadas uma vez que Terapia Cognitivo Comportamental, pode auxiliar esses sujeitos a terem mais autonomia, controlar suas ansiedades e conviver melhor na sociedade em que estão inseridos. Observou-se no decorrer deste estudo que as características das pessoas autistas muitas vezes podem ser confundidas com outros transtornos, daí a demora no fechamento do diagnóstico, por isso é importante que as observações sejam perspicaz tendo em vista que a pessoa com autismo já nasce com esse transtorno e não o adquire ao longo da vida, as características já são presentes desde a infância, e persistem por toda vida com quadros que podem mudar quanto ao aspectos sociais e comportamentais dependendo do estímulo que essas pessoas tem no meio em que vivem.

Conclui-se que há uma enorme variabilidade de sintomas do autismo, cada autista apresenta um comportamento diferente dependendo das estimulações que receberam, ora, um autista que foi acompanhado por médicos e equipe multiprofissional desde a infância, recebendo todos os estímulos, chega a juventude e fase adulta sem grande comprometimento nos aspectos sociais, de comunicação e cognição. Dado exposto considera-se que quando o diagnóstico é feito no período considerado como o mais adequado, os resultados das intervenções de modo geral tendem a ser mais promissores. 
Vale ressaltar que as literaturas que abordam a importância da TCC para o auxílio a pacientes autistas com diagnostico tardio ainda são escassas sendo necessário que mais trabalhos articulem a importância da TCC nesse contexto. Dessa forma propõem-se que mais pesquisas sejam realizadas nessa área intuindo o melhor desenvolvimento em todas as esferas da pessoa com autismo diagnosticado tardiamente.

A titulo conclusivo, espera-se que este estudo contribua com profissionais de saúde, educação e social uma vez que em se tratando do sujeito com TEA, considera-se importante o dialogo multidisciplinar entre todos os envolvidos no acompanhamento do portador desse transtorno a fim de proporcionar um processo de adaptação mais positiva na sociedade. Considera-se importante destacar que a relação dos profissionais com a família é fundamental no processo de atendimento, acompanhamento e desenvolvimento do autista pois, podem trazer avanços no campo social, educação e de inclusão.

\section{REFERÊNCIAS}

AGUIAR, Camila Stor. Baralho do modelo cognitivo para adultos: psicoeducação dos pensamentos, sentimentos e comportamentos. Novo Hamburgo: Sinopsys, 2018.

ASSOCIAÇÃO PSIQUIÁTRICA AMERICANA. Manual Diagnóstico e Estatístico de Transtornos Mentais, Quinta Edição (DSM-V):Arlington, VA: Associação Americana de Psiquiatria, 2013.

\section{ASSOCIAÇÃO PSIQUIÁTRICA AMERICANA. Manual Diagnóstico e Estatístico de} Transtornos Mentais, Quinta Edição (DSM-V). Arlington, VA: Associação Americana de Psiquiatria, 2013.

BAHLS, S. C. \& Navolar, A. B. B. Terapia cognitivo-comportamentais: conceitos e pressupostos teóricos. Psico UTP Online, ㄲo 04, 2010. Disponível em: $<$ http://www.utp.br/ psico.utp.online/site4/terapia_cog.pdf >. Acesso em 16 de abril de 2020. 
BAIO J, Lazarus C, Autry A, Avchen RN, Van Naarden Braun K. Impact of postcensal versus intercensal population estimates on prevalence of selected developmental disabilities metropolitan. Atlanta, Georgia, 1991-1996. Am J Ment Retard 2009;112:462-6.

BAIO, J. et al. Prevalência de Transtorno do Espectro do Autismo entre adultos e adolescentes - Rede de Monitoramento do Autismo e Deficiências do Desenvolvimento, Estados Unidos, 2014.

BAPTISTA CLÁUDIO ROBERTO, Cleonice Bosa. Autismo e educação: Reflexões propostas de intervenção. Porto Alegre, Artmed, 2002.

BARON COHEN, Lai, M. Identificando a geração perdida de adultos com condições do espectro do autismo. Departamento de Psiquiatria, Universidade de Cambridge, Cambridge, Reino Unido, 2015.

BECK, J. Terapia cognitivo-comportamental: teoria e prática. Porto Alegre: Artmed, 2014.

BhAtTACHARJEE, D., Rai, A. K., Singh, N. K., Kumar, P., Munda, S. K., \& Das, B. Psychoeducation: A measure to strengthen psychiatric treatment. Delhi Psychiatric Journal, 14(1),33-39. 2016.

BORBA, M. M. C.; BARROS, R. S. Ele é autista: como posso ajudar na intervenção? Um guia para profissionais e pais com crianças sob intervenção analíticocomportamental ao autismo. Cartilha da Associação Brasileira de Psicologia e Medicina Comportamental (ABPMC), 2018.

BORGES, Manuela e SHINOHARA, Helene. Síndrome de Asperger em paciente adulto: um estudo de caso. Rev. bras.ter. cogn. 2013, vol.8, n.1, pp. 41- 48. ISSN 1982-3746.

BRITES, Clay. Mentes únicas - São Paulo: Editora Gente, 2019. 
CAIANA, Fred; REICHOW, Brian; MCPARTLAND, James. Adultos com distúrbios do espectro do autismo. Nova York, Springer, 2016.

CDC- CENTRO DE PREVENÇÃO E CONTROLE DE DOENÇAS. Departamento de Saúde e Serviços Humanos dos EUA. Biossegurança em Laboratórios Biomédicos e de Microbiologia. 15a edição. Washington. EUA. 2014. Tradução: Ministério da Saúde. Fundação Nacional de Saúde. Brasília, DF. 2016.

CERVO, A. L.; BERVIAN, P. A. Metodologia científica. São Paulo: Prentice Hall, 2013.

DIAS SANDRA. Asperguer e sua síndrome em 1944 e na atualidade. Rev. Latino americana. vol.18 no.2 São Paulo jun. 2015.

FECTEAU S, Mottron L, Berthiaume $\mathrm{C}$, et al. Mudanças no desenvolvimento de sintomas autistas. Autismo. Setembro de 2017; 7 (3): 255-68.

FONSECA, Maria Elisa Granchi; CIOLA, Juliana de Cássia Baptistella. O programa TEACCH: estrutura e formas de aplicação na realidade brasileira. Pirassununga, v.1, 2009.

GADIA, Carlos. Aprendizagem e autismo: transtornos da aprendizagem: abordagem neuropsicológica e multidisciplinar. Porto Alegre: Artmed, 2014.

GAUDERER, C. Autismo e outros atrasos do desenvolvimento - Guia Prático Para Pais e Profissionais. 14ํe ed. revista e ampliada. Revinter, 2014.

GIL ANTÔNIO CARLOS. Como elaborar projetos de pesquisa, 6ํed. Atlas, 2017.

GUPTA, A. \& State, Autismo: genética. Revista Brasileira de Psiquiatria, 28, 29-38, 2006.

HESSELMARK, Eva, Stephanie e Bejerot, Susanne. Grupo cognitivo terapia comportamental e atividade recreativa em grupo para adultos com autismo: Um estudo clínico randomizado preliminar. Autism 2014. Vol. 18 (6) 672-683. 
KANNER, L. Affective disturbances of affective contact. The Nervous Child, 2, 217 - 250. 1943.

KNAPP, BECK. Princípios fundamentais da terapia cognitiva. Terapia cognitivocomportamental na prática psiquiátrica (pp. 19-41). Porto Alegre. Artmed, 2011.

KWEE, Caroline Sianlian; Marinho Tania Maria; Atherino, Ciríaco Cristóvão Tavares. Autismo: uma avaliação transdisciplinar baseada no programa TEACCH. Rev. CEFAC, São Paulo, v. 11, supl. 2, p. 217-226, 2018.

LIMA, Maria Cecília \& Dilascio. Mariana. Treinamento de habilidades sociais na Síndrome de Asperger. Rev debates em psiquiatria. Jan/fev 2016. Disponível em < http://abp.org.br/rdp16/01/RDP_1_201602.pdf> acesso em 14 de Abril 2 de 2020.

LINHARES, M. B. M., Carvalho, A. E. V., Padovani, F. H. P., Bordin, M. B., Martins, I. M. B., \& Martinez, F. E. Compreensão do fator de risco da prematuridade sob a ótica desenvolvimental. São Paulo, 2012. Casa do Psicólogo.

LOVAAS, O. Ivas; SMITH, Tristam. Una teoria conductual comprehensiva del autismo como paradigma para investigación y tratamiento. 2010 Disponível em <http://www.comportamental.com>. Acesso em 23 março de 2020.

MELLO, A.M. S. R. \& Vatavuk, M.C. Autismo: Guia Prático. 5 ed. São Paulo: AMA; Brasília: Corde, 2007.

MIZAEL TÁHCITA MEDRADO; Ana Lúcia Rossito Aiello. Revisão de estudos sobre o Picture Exchange Communication System (PECS) para o ensino de linguagem a indivíduos com autismo e outras dificuldades de fala. Rev. bras. educ. espec. vol.19 no.4 Marília Oct./Dec. 2013.

MONIZ, A. A terapia Cognitivo Comportamental e seus benefícios. Universidade do Minho; 2007.

ORGANIZAÇÃO MUNDIAL DA SAÚDE. Classificação Estatística Internacional de Doenças e Problemas Relacionados à Saúde: CID-10 Décima revisão. Trad. do 
Centro Colaborador da OMS para a Classificação de Doenças em Português. 3 ed. São Paulo: EDUSP; 1996.

ORGANIZAÇÃO PAN-AMERICANA DA SAÚDE. OMS divulga nova Classificação Internacional de Doenças (CID 11). Brasília, DF: OPAS, 2018. Disponível em: https://www.paho.org/bra/index.php?option=com_content\&view=article\&id=5702:oms divulga-nova-classificacao-internacional-de-doencas-cid-11\&ltemid=875. Acesso em 15 de setembro de 2020.

RAMOS, J, Xavier, S, Morins. Perturbações do espectro do autismo no adulto e suas comorbilidades psiquiátricas. Psilogos 10(2): 9-23. American Psychiatric Association 2014.

REICHOW, B. Overview. Of meta-analyses on early intensive behavioral intervention for young children with autism spectrum disorders. Journal of Autism and Development Disorders, 42(4), 512-520, 2012.

ROBERTSON, S. M. Neurodiversity, quality of life, and autistic adults: shifting research and professional focuses onto real-life challenges. Disability Studies Quarterly, v. 30, n. 1, 2015.

SAMPAIO. L. Um estudo preliminar sobre a construção da comunicação com autistas. XII Simpósio Brasileiro Autismo e Seus Desafios. Goiânia, 2005.

SANTOS, Ana Maria Tarcitano. Autismo: desafios na escolarização de adultos autistas. Centro de Referências em Distúrbios de Aprendizagem. São Paulo: CRDA, 2017.

SELTZER MM, Shattuck $P$, Abbeduto $L$, et al. Trajetória de desenvolvimento em adolescentes e adultos com autismo. Ment Retard Dev Disabil Res Rev. 10 (4): 234-47, 2017.

SERRA, Felipe. Desafios e possibilidades para a pessoa com autismo. São Leopoldo: Pandorga, 2014. 
SILVA, Micheline and MULICK, James. Diagnosticando o transtorno autista: aspectos fundamentais e considerações práticas. Psicol. cienc. prof. [online]. 2009, vol.29, n.1, pp.116-131. ISSN 1414-9893.

SILVEIRA, José Carlos. Atuação do psicólogo frente aos transtornos globais do desenvolvimento. Psicol. cienc. prof., Brasília, v. 24, n. 2, June 2015.

SOARES, L. Programa de Apoio Pedagógico e inclusão: um estudo de caso com autistas. Revista da ABEM, Londrina. v. 20 n. 27, p. 55-64, 2012.

UNG, D., Selles, R., Small, B. J., \& Storch, E. A. (2015). A systematic review and meta-analysis of cognitive-behavioral therapy for anxiety in youth with highfunctioning autism spectrum disorders. Child Psychiatry \& Human Development, 46(4), 533-547.

WHIT, Thomas. O desenvolvimento do autismo. São Paulo: M. Books, 2013.

ZEEDYK M. Suzanne, Phoebe Caldwell e Clifford E. Davies. Com que rapidez a Interação Intensiva promove o engajamento social para adultos com profundas dificuldades de aprendizagem? European Special Needs Education, 24: 2, 119-137, 2010.

Enviado: Outubro, 2020.

Aprovado: Dezembro, 2020. 